\title{
Mitotic replisome disassembly depends on TRAIP ubiquitin ligase activity
}

\author{
Sara Priego Moreno*, Rebecca M Jones*, Divyasree Poovathumkadavil, Shaun Scaramuzza, Agnieszka Gambus (1)
}

\begin{abstract}
We have shown previously that the process of replication machinery (replisome) disassembly at the termination of DNA replication forks in the S-phase is driven through polyubiquitylation of one of the replicative helicase subunits $(\mathrm{Mcm} 7)$ by $\mathrm{Cul}^{\mathrm{LRR} 1}$ ubiquitin ligase. Interestingly, upon inhibition of this pathway in Caenorhabditis elegans embryos, the replisomes retained on chromatin were unloaded in the subsequent mitosis. Here, we show that this mitotic replisome disassembly pathway exists in Xenopus laevis egg extract and we determine the first elements of its regulation. The mitotic disassembly pathway depends on the formation of K6- and K63-linked ubiquitin chains on Mcm7 by TRAIP ubiquitin ligase and the activity of p97/VCP protein segregase. Unlike in lower eukaryotes, however, it does not require SUMO modifications. Importantly, we also show that this process can remove all replisomes from mitotic chromatin, including stalled ones, which indicates a wide application for this pathway over being just a "backup" for terminated replisomes. Finally, we characterise the composition of the replisome retained on chromatin until mitosis.
\end{abstract}

DOI 10.26508/lsa.201900390 | Received 26 March 2019 | Revised 28 March 2019 | Accepted 29 March 2019 | Published online 12 April 2019

\section{Introduction}

Faithful cell division is the basis for the propagation of life and requires accurate duplication of all genetic information. DNA replication must be precisely regulated as unrepaired mistakes can change cell behaviour with potentially severe consequences, such as genetic disease, cancer, and premature ageing (Burrell et al, 2013). Fundamental studies have led to a step change in our understanding of the initiation of DNA replication and DNA synthesis, but until discovery of the first elements of the eukaryotic replisome disassembly mechanism in 2014 (Maric et al, 2014; Moreno et al, 2014), the termination stage of eukaryotic replication was mostly unexplored.

DNA replication initiates from thousands of replication origins. They are the positions within the genome where replicative helicases become activated and start unwinding DNA while moving in opposite directions, away from each other, creating two DNA replication forks.
The replicative helicase is composed of $\mathrm{Cdc} 45, \mathrm{Mcm} 2-7$ hexamer, and GINS complex (CMG complex) (Moyer et al, 2006); it is positioned at the tip of replication forks and forms a platform for replisome assembly (Replisome Progression Complex) (Gambus et al, 2006). Once established, the replication forks replicate chromatin until they encounter forks coming in opposite directions from neighbouring origins. At this point, termination of replication forks takes place. As CMG helicases travel on the leading strand templates at the forks, the strand encircled by converging helicases differs because of the antiparallel nature of the DNA molecule (Fu et al, 2011). The two converging helicases can therefore pass each other, allowing for completion of DNA synthesis. Finally, removal of the replisome from fully duplicated DNA is the last stage of termination of forks (Dewar et al, 2015). We have shown that in Xenopus laevis egg extract and in Caenorhabditis elegans embryos, this replisome removal in S-phase is driven by Cul2 ${ }^{\text {LRR }}$ ubiquitin ligase, which ubiquitylates Mcm7 within the terminated CMG complex (Sonneville et al, 2017). Such modified CMG is then recognised by p97/VCP segregase and removed from chromatin allowing for disassembly of the whole replisome built around the helicase (Moreno et al, 2014).

Most notably, we have shown that in C. elegans embryos, any helicase complexes that fail to be unloaded in the S-phase are alternatively unloaded in the prophase of mitosis (Sonneville et al, 2017). This potential backup mechanism can be detected when CUL-2 ${ }^{\text {LRR-1 }}$ activity is blocked and, like S-phase pathway, depends on the p97 segregase for unloading. Unlike the S-phase pathway, however, it requires an additional p97 cofactor UBXN-3/FAF1 and the SUMO-protease ULP-4 (Senp6/7 homologue in higher eukaryotes) (Sonneville et al, 2017). Interestingly, budding yeast do not possess this mitotic replisome disassembly pathway; cells lacking SCF ${ }^{\text {Dia2 }}$ activity, the ubiquitin ligase responsible for $\mathrm{Mcm} 7$ ubiquitylation in Saccharomyces cerevisiae, accumulate posttermination replisomes on DNA until the next G1 of the next cell cycle (Maric et al, 2014). Our aim, therefore, was to determine if this mitotic replisome disassembly pathway is functioning in higher eukaryotes or if it is a phenomenon specific to C. elegans embryos. Here, we show that a mitotic replisome disassembly pathway does exist in $X$. laevis egg extract and determine the first elements of its

Institute for Cancer and Genomic Sciences, College of Medical and Dental Sciences, University of Birmingham, Birmingham, UK

Correspondence: a.gambus@bham.ac.uk

Sara Priego Moreno's present address is Salk Institute for Biological Studies, La Jolla, CA, USA

*Sara Priego Moreno and Rebecca M Jones contributed equally to this work 
regulation. We show that only a restricted part of the replisome stays retained on chromatin through into mitosis in Xenopus egg extract. The disassembly of this replisome is independent of Cullintype ubiquitin ligases but requires p97 segregase function. Mitotic replisome disassembly depends on K6- and K63-linked ubiquitin chains but not SUMO modifications. In addition, we show that stalled forms of helicase can also be unloaded using the same mechanism, suggesting that rather than being a backup pathway for the disassembly of terminated replisomes, this process is essential to remove any replisome from chromatin before cell division. Finally, we identify TRAIP ubiquitin ligase as essential for Mcm7 ubiquitylation and replisome disassembly in mitosis.

\section{Results}

$X$. laevis egg extract is a cell-free system, which has proven to be instrumental over the years in studies of DNA replication. Xenopus egg extract contains stockpiles of cell cycle factors which support efficient replication of DNA templates in vitro, with the recapitulation of most of the biochemical reactions that take place in living cells. To retain high synchronicity in our system, we restrict the replication reaction in the extract to only one round through blocking protein synthesis with cycloheximide, which blocks cyclins production and progression of extract into mitosis (Gillespie et al, 2012). However, to determine the existence of a mitotic replisome disassembly pathway in Xenopus egg extract we needed to allow for this progression. To achieve this, we supplemented the extract with recombinant cyclin after completion of DNA replication. Xenopus egg extract synthesises cyclin A1 (embryonic form of cyclin A), B1, and B2 (Minshull et al, 1989). Whereas the B family of cyclins has been shown to drive Xenopus meiotic division and oocyte maturation (Hochegger et al, 2001), both cyclin A and B have been shown to promote egg extract transition to mitosis (Strausfeld et al, 1996). We therefore purified His-tagged $X$. laevis cyclin A1 N $\triangle 56$ (hereafter: cyclin $A 1 \Delta$ ) and added it to the extract upon completion of DNA replication, as described previously, to induce mitotic entry (Strausfeld et al, 1996). The N-terminal deletion to cyclin A1 prevents its degradation and ensures that the extract remains arrested in mitosis, reducing de-synchronisation of our experiments. In all of the experiments described below, we supplemented extract with cyclin A1 $\triangle$ after completion of DNA replication. As a result, addition of cyclin A1 $\triangle$ did not stimulate any more DNA synthesis (Fig S1A), but it did lead to progression into mitosis, as evidenced by breakage of the nuclear envelope, condensation of chromatin into chromosomes, and phosphorylation of Serine 10 on histone $\mathrm{H} 3$, which coincides with chromosome condensation (Fig S1B and C). Moreover, we could detect chromatin binding of condensin Smc2-another clear sign of the mitosis stage (Fig S1C).

To test if the replisome, which is retained on chromatin in S-phase, can be unloaded as cells enter mitosis, we needed to inhibit S-phase replisome disassembly. To achieve this, a replication reaction was set up in the interphase extract supplemented with Cullin ligase inhibitor MLN4924 to block Cul2 ${ }^{\text {LRR1 }}$ activity (Sonneville et al, 2017). Addition of MLN4924 to egg extract did not affect its ability to synthesise DNA as shown previously (Moreno et al, 2014) and in Fig S1A. Moreover, the timing of replication completion was very reproducible within a batch of extract (Fig S1A). Throughout this article, we confirmed the timing of replication completion for every extract used. To do this, we determined the time point, after addition of sperm DNA, when no more ${ }^{32} \mathrm{P}$-labelled dATP was incorporated into DNA. At this point, the components of the replisome were also seen to be unloaded from chromatin in the control samples, but retained on chromatin in those samples supplemented with Cullin ligase inhibitor MLN4924 (Fig S1D). We then optionally added cyclin A1 $\Delta$ at the replication completion time (usually 90 min after sperm DNA addition), isolated chromatin at different time points during mitosis progression, and analysed chromatin-bound proteins by Western blotting (Fig $1 \mathrm{~A}$ and B). The presence of the Cullin ligase inhibitor MLN4924 in the S-phase extract did not affect the DNA synthesis level nor induction of mitosis in our extract (Fig S1A and C). As seen in Fig 1B, in control samples without inhibition of replisome disassembly in S-phase, there were no CMG helicase components (hereafter represented by Cdc45 and Psf2 subunits) associated with chromatin at any times analysed, as replisome disassembly takes place before addition of cyclin A1 1 . Notably, we did detect low levels of PCNA bound to chromatin in late $\mathrm{S}$-phase and mitosis as it remains on DNA after Okazaki fragment maturation and completion of replication, so as to aid post-replicative DNA repair (Gao et al, 2017). When replisome disassembly in S-phase was blocked with MLN4924 treatment, the CMG helicase remained associated with chromatin, as expected, and Mcm7 displayed low levels of ubiquitylation. Similar low levels of $\mathrm{Mcm} 7$ ubiquitylation have been shown previously upon both MLN4924 treatment and Cul2 immunodepletion (Moreno et al, 2014; Sonneville et al, 2017) and could indicate residual activity of the Cul2 ${ }^{\mathrm{LRR} 1}$ ligase or activity of yet another unidentified ligase. Importantly, upon addition of cyclin A1 $\triangle$, Cdc45, Psf2, and ubiquitylated Mcm7 were efficiently unloaded (Fig 1B). This result indicates that indeed the mitotic replisome disassembly pathway is evolutionarily conserved and that, unlike the S-phase pathway, it does not require the activity of Cullin-type ubiquitin ligases because the Cullin ligase inhibitor MLN4924 was present throughout the reaction. The continuous presence of unmodified $\mathrm{Mcm} 7$ in our samples is a result of the high quantity of DNA used in our experiments. This allows us to clearly detect the replication fork components and ubiquitylated $\mathrm{Mcm} 7$ by Western blotting. Because of the high quantity of DNA used, some of the nuclei were not able to form completely and failed to initiate replication, resulting in the isolation of unfired Mcm2-7 complexes, as shown previously (Moreno et al, 2014). However, when we added a much lower quantity of DNA and used minimal licensing conditions (addition of recombinant geminin, 2 min after sperm DNA), we could detect unloading of $\mathrm{Mcm} 7$ in mitosis together with Cdc45 and Psf2 (Fig S2A).

Next, we tested whether the mitotic replisome disassembly pathway requires the activity of the p97 segregase. We followed the experimental setup as before but now optionally added the inhibitor of p97, NMS873, along with cyclin A1 $\triangle$ to inhibit p97 activity during mitosis. Addition of NMS873 to the extract together with cyclin A1 $\triangle$ did not affect extract transition to mitosis (Fig S2B). In these conditions, the retained replisome was unloaded upon cyclin A1 $\Delta$ addition in the absence but not in the presence of the p97 
A
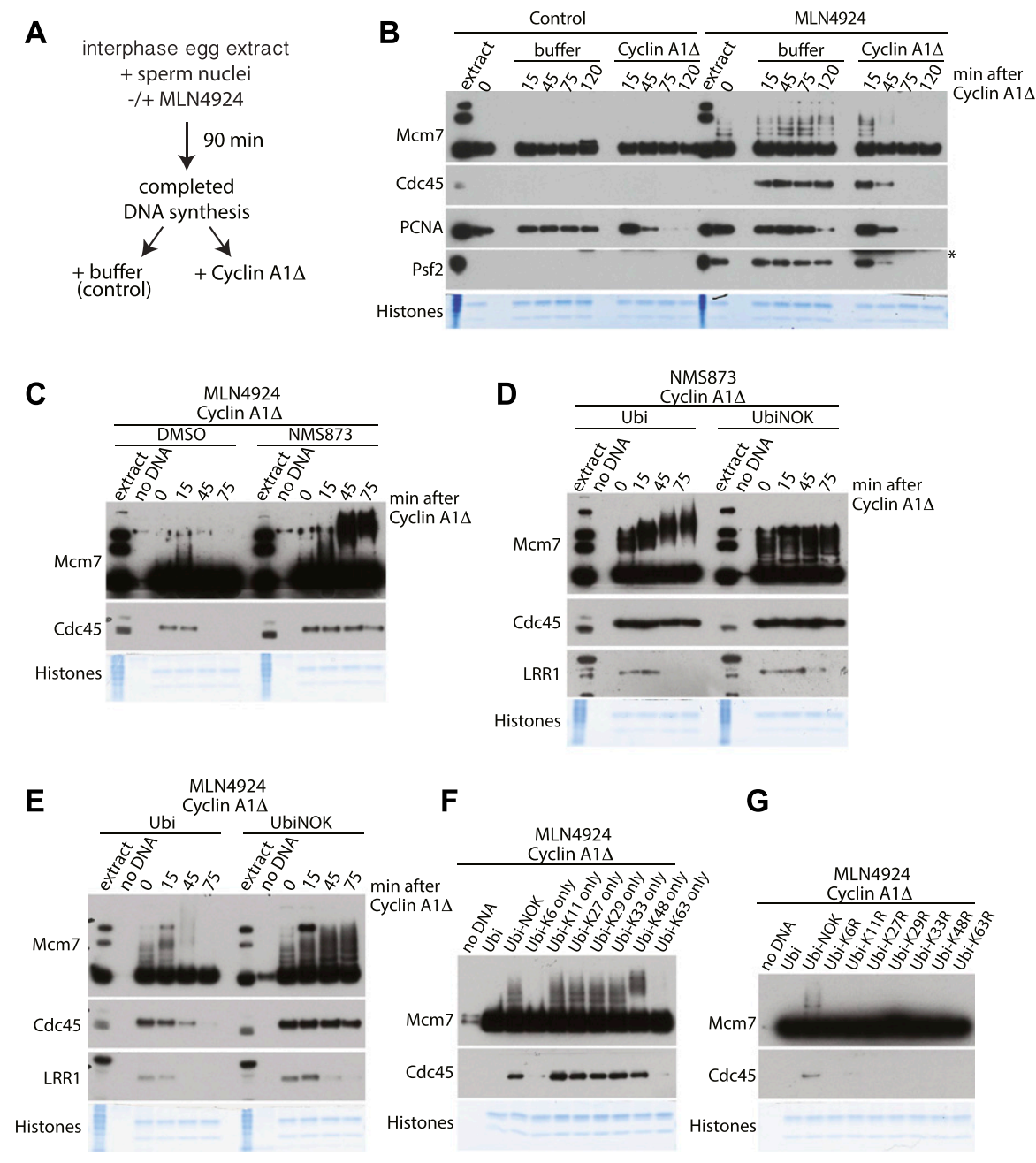

\section{$\mathbf{F}$}

G

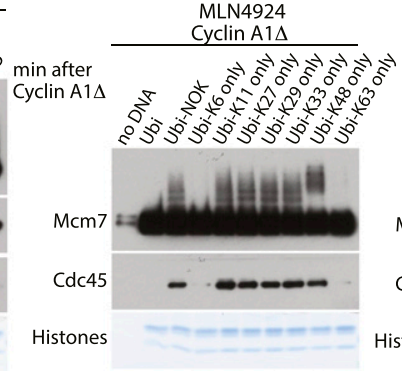

Figure 1. Mcm7 is ubiquitylated with $\mathrm{K} 6$ and $\mathrm{K} 63$ ubiquitin chains in mitosis and removed from chromatin by $\mathrm{p} 97$ segregase.

(A) Experimental design for driving egg extract into mitosis. (B) Experiment following design in (A). DNA was replicated to completion (90 $\mathrm{min}$ ) in egg extract supplemented optionally with a Cullin ligase inhibitor MLN4924. After completion of the replication reaction, cyclin A1 $\Delta$ was optionally added to the extract to drive extract into mitosis. Chromatin was isolated at indicated time points after cyclin A1 $\Delta$ addition and chromatin samples analysed by Western blotting with the indicated antibodies. Time "0" sample was isolated at the replication completion time when cyclin A $1 \Delta$ was added to the extract. Colloidal Coomassie-stained histones serve as a quality and loading control. An asterisk "*" by the Psf2 blot indicates a band of cyclin $\mathrm{A} 1 \Delta$ that is recognised by Psf2 antibody. (C) The replication reaction was completed in the presence of Cullin ligase inhibitor MLN4924 and driven into mitosis by addition of cyclin A1 $\Delta$. At the same time as cyclin A1 $\triangle$ half of the sample was supplemented additionally with p97 inhibitor NMS873. Chromatin samples were isolated at indicated time points and analysed as in (B). A sample without DNA addition (no DNA) was processed alongside others as a chromatin specificity control. (D) The replication reaction was completed in the presence of p97 inhibitor NMS873 and driven into mitosis by addition of cyclin A1 $\Delta$. At the same time as cyclin A1 $\Delta$, the samples were supplemented with recombinant wt ubiquitin or UbiNOK. Chromatin samples were analysed as above. (E) Experiment as in (D) but replication reaction was carried out in the presence of Cullin ligase inhibitor MLN4924 instead of p97 inhibitor NMS873. (F, G) Replication reaction was completed in the presence of Cullin ligase inhibitor MLN4924 and driven into mitosis by addition of cyclin $\mathrm{A} 1 \Delta$. At the same time as cyclin $\mathrm{A} 1 \Delta$ addition, the extract was supplemented with the indicated mutants of ubiquitin. Chromatin was isolated at 75 min after cyclin A1 $\Delta$ addition and analysed by Western blotting as above.

Source data are available for this figure. inhibitor, indicating that indeed p97 does play an essential role in promoting mitotic replisome disassembly (Fig 1C). We could also see an analogous result if the p97 inhibitor was present throughout the two stages of the cell cycle as the only way to block replisome disassembly (Fig S2C). Importantly, the presence of p97 inhibitor NMS873 throughout interphase does not impede DNA synthesis (Sonneville et al, 2017) and does not induce additional DNA synthesis when combined with addition of cyclin A1 $\triangle$ (Fig S2D).

Interestingly, when mitotic unloading of replisome was blocked with p97 inhibitor, we could clearly see accumulation of highly modified forms of Mcm7 on chromatin (Figs 1C and S2C). To examine whether these modifications were due to further ubiquitylation of Mcm7 in mitosis, we blocked S-phase and mitotic replisome disassembly by addition of $\mathrm{p} 97$ inhibitor from the beginning of the replication reaction, induced mitosis after completion of DNA synthesis, and optionally supplemented extract with a high concentration of wt ubiquitin (Ubi) or a chain-terminating mutant of ubiquitin with all lysines mutated (UbiNOK). Supplementation of extract with UbiNOK at the same time as cyclin A1 $\triangle$ addition allowed for normal entry into mitosis as indicated by nuclear envelope breakdown (Fig S2E). Addition of Ubi allowed for accumulation of highly modified Mcm7 on chromatin in mitosis as before (Fig 1D), but
UbiNOK blocked further modifications of Mcm7, leaving only the chains which were built previously in S-phase (Fig 1D). To determine whether this further Mcm7 polyubiquitylation in mitosis is essential for mitotic replisome disassembly, we repeated the experiment with addition of wt Ubi or UbiNOK, but this time only in the presence of the Cullin ligase inhibitor MLN4924 from the start of the reaction (Fig 1E). Indeed, addition of UbiNOK to mitotic extract did block disassembly of the replisome (as shown by permanent Cdc45 chromatin binding), suggesting that further Mcm7 polyubiquitylation is required for mitotic replisome unloading. We also observed that LRR1 (the substrate-specific subunit of Cullin 2, targeting $\mathrm{Mcm} 7$ in S-phase) dissociates from chromatin in mitosis irrespectively of replisome disassembly, in agreement with the finding that it does not play an essential role in this pathway (Fig 1D and E). Importantly, these results indicate that a previously unreported ubiquitin ligase is needed for $\mathrm{Mcm} 7$ ubiquitylation and replisome disassembly in mitosis.

As the ubiquitin ligase acting in the mitotic pathway differed from that of the S-phase pathway, we decided to test whether the type of ubiquitin chains built on Mcm7 in mitosis also differed. To determine which ubiquitin chains are required for mitotic $\mathrm{Mcm} 7$ ubiquitylation and replisome disassembly, we supplemented 
extract with Cullin ligase inhibitor MLN4924, allowed for completion of DNA synthesis, and subsequently induced mitosis along with addition of a series of ubiquitin mutants that have only one lysine left in their sequence (Fig 1F). We observed that only wt ubiquitin and ubiquitin containing only lysine 6 ( $\mathrm{K} 6$ only) or lysine 63 (K63 only) could support mitotic replisome disassembly (as visualised by the absence of Cdc45 on chromatin at 75 min after inducing mitosis) (Fig 1F). Interestingly, chains linked through lysine 48 (K48), which are responsible for S-phase unloading (Moreno et al, 2014), could still be attached to Mcm7 in mitosis (upshift of modified Mcm7 forms), but they could not support unloading of the replisome as Cdc45 remained associated with chromatin. In a reciprocal experiment, we used a series of ubiquitin mutants with only one of the lysines within ubiquitin mutated (Fig 1G). All of the mutants used, apart from the UbiNOK control mutant, supported disassembly of the replisome, suggesting that either $\mathrm{K} 6$ or $\mathrm{K} 63$ can fulfill the mitotic pathway requirements (Fig 1G).

Having established that the type of ubiquitin chains and the type of ubiquitin ligase used by the mitotic pathway of replisome disassembly were different to those acting in the S-phase pathway, our aim was to identify this ubiquitin ligase. To this end, we decided to immunoprecipitate the replisome retained on mitotic chromatin and analyse all the interacting proteins by mass spectrometry. We set up a replication reaction in the presence of caffeine and the p97 inhibitor NMS873 and induced mitosis upon completion of DNA synthesis (90 min). Neither of the treatments affected the extract's ability to synthesise DNA (Fig S3A). We then immunoprecipitated $\mathrm{Mcm} 3$ from mitotic chromatin and analysed the interacting factors by mass spectrometry. First, we determined which components of the replisome are still retained on chromatin in mitosis. For this, we compared the replisome components retained on chromatin in mitosis with S-phase post-termination replisome, reported previously (Sonneville et al, 2017) (Fig 2A and B). Interestingly, although inhibition of replisome disassembly in the S-phase led to accumulation of the whole replisome on chromatin (Sonneville et al, 2017), only a selection of replisome components stayed on chromatin in mitosis. All of the lagging strand components of the replisome were lost, as were $\mathrm{Mcm} 10$ and Claspin, whereas levels of Ctf4/And-1, Timeless, Tipin, and Pol epsilon were also reduced (Fig $2 \mathrm{~A}$ and $\mathrm{B})$. This suggests that only components directly interacting with the CMG remained accumulated around it through to mitosis, whereas others, more peripheral to $C M G$, could dissociate over time.

The level of histone chaperone FACT (Spt16 and SSRP) stayed the same between S-phase and mitosis. This suggests that the retained replisome in mitosis has the potential ability to move through chromatin as FACT is likely to displace nucleosomes in front of such a replisome. We could see also that Cul2 ${ }^{\mathrm{LR} 1}$, which strongly accumulated in the S-phase post-termination replisome, is not a major component of the mitotic replisome, as expected from previous data (Fig 1D and E).

Finally, we detected two other ubiquitin ligases interacting with the mitotic helicase: TRAIP and RNF213. More specifically, we found that TRAIP interacts with the post-termination replisome in S-phase, but it is enriched in mitosis, whereas RNF213 is a minor interactor of only the mitotic replisome (Fig 2A). The TNFreceptor-associated factor (TRAF)-interacting protein (TRAIP, also known as TRIP or RNF206) was originally identified through its ability to bind TRAF1 and TRAF2 and shown to inhibit NFkB activation (Lee et al, 1997). It has been since shown that TRAIP is an E3 ubiquitin ligase, which is essential for cell proliferation (Besse et al, 2007; Park et al, 2007), and which is required for resolution of replication stress (Feng et al, 2016; Harley et al, 2016; Hoffmann et al, 2016) and for regulation of the spindle assembly checkpoint during mitosis (Chapard et al, 2014). TRAIP is ubiquitously expressed, with its expression regulated by E2F transcription factors and protein stability controlled by the ubiquitin proteasome pathway-as a result, the protein level of TRAIP peaks in the G2/M stage of the cell cycle (Chapard et al, 2015). On the other hand, RNF213 (mysterin) is a large (591 kD) ATPase/E3 ligase, which is mostly known as being a susceptibility gene for moyamoya disease (cerebrovascular disease) (Kamada et al, 2011; Liu et al, 2011). Of note, RNF213 ${ }^{-/-}$mice do not show any apparent health problems (Kobayashi et al, 2013; Sonobe et al, 2014) and more recently, RNF213 was shown to globally regulate ( $\alpha$-ketoglutarate)-dependent dioxygenases ( $\alpha$-KGDDs) and non-mitochondrial oxygen consumption (Banh et al, 2016). To support our mass spectrometry data, we tested a number of antibodies by Western blotting against RNF213 and TRAIP to confirm their association with the chromatin-bound replisome in mitosis. Although we were unsuccessful with detection of any signal for RNF213, we were able to show that TRAIP interacts with the replisome retained on chromatin in mitosis (Figs $2 \mathrm{C}$ and $\mathrm{S3B}$ ).

After confirming that the ubiquitin ligase TRAIP is the likely candidate responsible for $\mathrm{Mcm} 7$ ubiquitylation and replisome disassembly in mitosis, we next characterised TRAIP chromatin binding dynamics during the two cell cycle stages and the replisome disassembly process. We found that although TRAIP associated weakly with the S-phase chromatin at times when forks progress through chromatin and replicate DNA, it accumulated strongly on the S-phase chromatin upon inhibition of replisome disassembly with the p97 inhibitor NMS873 (Fig 3A). Importantly, TRAIP also accumulated on mitotic chromatin when replisome disassembly was inhibited with the p97 inhibitor, following the same pattern as replisome components (Fig 3B). To test whether TRAIP is indeed the ubiquitin ligase responsible for unloading of replisome in mitosis, we aimed to inhibit TRAIP enzymatic activity in our extract. As we were unable to efficiently immunodeplete TRAIP from the egg extract with any of the antibodies tested, we decided to use a dominant-negative, ligase-dead mutant of TRAIP to out-compete the endogenous TRAIP. To this end, we purified recombinant His/SUMO-tagged $X$. laevis TRAIP, both wt and the C25A RING domain mutant, which has been shown to disrupt TRAIP ubiquitin ligase activity (Besse et al, 2007; Chapard et al, 2014) and (Fig S3C). We blocked disassembly of the replisome in S-phase by addition of the Cullin ligase inhibitor MLN4924 and drove extract into mitosis by addition of cyclin A1 $\triangle$, when we added recombinant wt or mutant TRAIP. Addition of neither wt nor mutant TRAIP affected the extract's ability to enter mitosis upon cyclin A1 $\triangle$ addition, as shown through nuclear envelope breakdown (Fig S3D) and Smc2 chromatin loading (Fig 3 C and D). As shown in Fig 3C, addition of the enzymatic dead mutant of TRAIP into mitotic extract inhibits unloading of post-termination replisomes retained on chromatin, whereas addition of wt TRAIP does not have such an effect. To confirm that enzymatic dead TRAIP indeed affects replisome 


\begin{tabular}{|c|c|c|c|c|c|}
\hline \multirow[t]{2}{*}{ A } & & \multicolumn{2}{|c|}{$\begin{array}{l}\text { IP from mitotic chromatin } \\
\text { with retained CMG (extract } \\
\text { treated with p97i) }\end{array}$} & \multicolumn{2}{|c|}{$\begin{array}{l}\text { IP from S-phase post-termination } \\
\text { chromatin with retained } \\
\text { replisome (Sonneville et al 2017) }\end{array}$} \\
\hline & Protein (kDa) & $\begin{array}{c}\text { IgG } \\
\text { TSC (coverage) }\end{array}$ & $\begin{array}{c}\alpha-M c m 3 \\
\text { TSC (coverage) }\end{array}$ & $\begin{array}{c}\text { IgG } \\
\text { TSC (coverage) }\end{array}$ & $\begin{array}{c}\alpha-M c m 3 \\
\text { TSC (coverage) }\end{array}$ \\
\hline 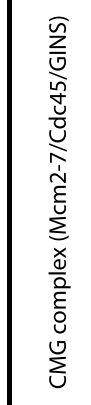 & $\begin{array}{c}\text { Mcm2 (100) } \\
\text { Mcm3 (90) } \\
\text { Mcm4 (97) } \\
\text { Mcm5 (82) } \\
\text { Mcm6 (93) } \\
\text { Mcm7 (82) } \\
\text { Cdc45 (66) } \\
\text { Psf1 (23) } \\
\text { Psf2 (21) } \\
\text { Psf3 (24) } \\
\text { Sld5 (26) }\end{array}$ & $\begin{array}{c}364(46 \%) \\
222(55 \%) \\
198(45 \%) \\
70(59 \%) \\
196(60 \%) \\
160(52 \%) \\
18(26 \%) \\
3(31 \%) \\
0 \\
3(15 \%) \\
2(10 \%)\end{array}$ & $\begin{array}{c}1348(78 \%) \\
1610(90 \%) \\
1277(85 \%) \\
720(88 \%) \\
964(91 \%) \\
1082(84 \%) \\
187(46 \%) \\
58(90 \%) \\
26(58 \%) \\
55(79 \%) \\
42(81 \%)\end{array}$ & $\begin{array}{c}17(17 \%) \\
17(20 \%) \\
7(7.4 \%) \\
30(17 \%) \\
39(14 \%) \\
24(8.9 \%) \\
2(2.6 \%) \\
0 \\
0 \\
0 \\
0\end{array}$ & $\begin{array}{c}975(71 \%) \\
1111(84 \%) \\
947(75 \%) \\
927(83 \%) \\
888(83 \%) \\
905(73 \%) \\
277(54 \%) \\
62(85 \%) \\
33(79 \%) \\
55(92 \%) \\
44(64 \%)\end{array}$ \\
\hline 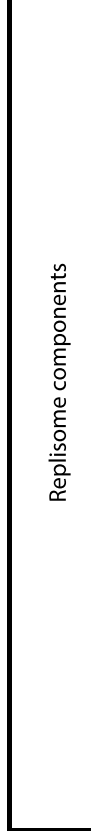 & 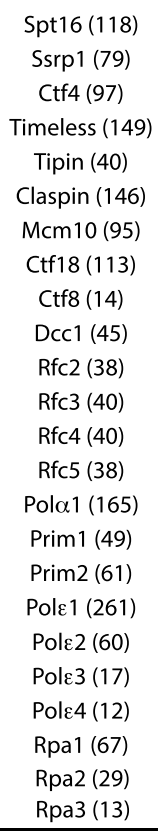 & $\begin{array}{c}109(23 \%) \\
19(8 \%) \\
4(4 \%) \\
6(3 \%) \\
0 \\
0 \\
0 \\
0 \\
0 \\
0 \\
0 \\
0 \\
0 \\
0 \\
0 \\
0 \\
0 \\
12(5 \%) \\
0 \\
0 \\
0 \\
80(53 \%) \\
13(22 \%) \\
6(52 \%)\end{array}$ & $\begin{array}{c}431(61 \%) \\
224(45 \%) \\
82(31 \%) \\
112(39 \%) \\
20(27 \%) \\
0 \\
0 \\
0 \\
0 \\
0 \\
0 \\
0 \\
0 \\
0 \\
0 \\
0 \\
0 \\
166(29 \%) \\
15(32 \%) \\
4(27 \%) \\
0 \\
107(48 \%) \\
30(55 \%) \\
10(92 \%)\end{array}$ & $\begin{array}{c}3(2.9 \%) \\
0 \\
0 \\
0 \\
0 \\
0 \\
0 \\
0 \\
0 \\
0 \\
0 \\
4(14 \%) \\
2(5.2 \%) \\
0 \\
0 \\
0 \\
0 \\
2(0.88 \%) \\
0 \\
0 \\
0 \\
0 \\
0 \\
0\end{array}$ & $\begin{array}{c}453(52 \%) \\
229(48 \%) \\
166(49 \%) \\
171(29 \%) \\
54(24 \%) \\
72(21 \%) \\
257(44 \%) \\
172(36 \%) \\
8(52 \%) \\
33(38 \%) \\
27(54 \%) \\
36(54 \%) \\
31(57 \%) \\
22(56 \%) \\
109(30 \%) \\
14(28 \%) \\
24(24 \%) \\
543(38 \%) \\
103(50 \%) \\
10(45 \%) \\
4(35 \%) \\
10(12 \%) \\
2(9 \%) \\
0\end{array}$ \\
\hline 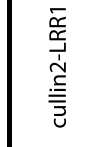 & $\begin{array}{l}\text { cullin2 (87) } \\
\text { LRR1 (47) } \\
\text { Elongin B (13) } \\
\text { Elongin C (12) }\end{array}$ & $\begin{array}{c}6(8 \%) \\
0 \\
0 \\
0\end{array}$ & $\begin{array}{c}4(3 \%) \\
11(21 \%) \\
0 \\
0\end{array}$ & $\begin{array}{l}0 \\
0 \\
0 \\
0\end{array}$ & $\begin{array}{c}124(52 \%) \\
46(44 \%) \\
5(31 \%) \\
7(32 \%)\end{array}$ \\
\hline 흑 & $\begin{array}{c}\text { Traip (87) } \\
\text { RNF213 (47) }\end{array}$ & $\begin{array}{c}2(4 \%) \\
0\end{array}$ & $\begin{array}{c}42(44 \%) \\
17(2 \%)\end{array}$ & $\begin{array}{l}0 \\
0\end{array}$ & $\begin{array}{c}34(36 \%) \\
0\end{array}$ \\
\hline
\end{tabular}
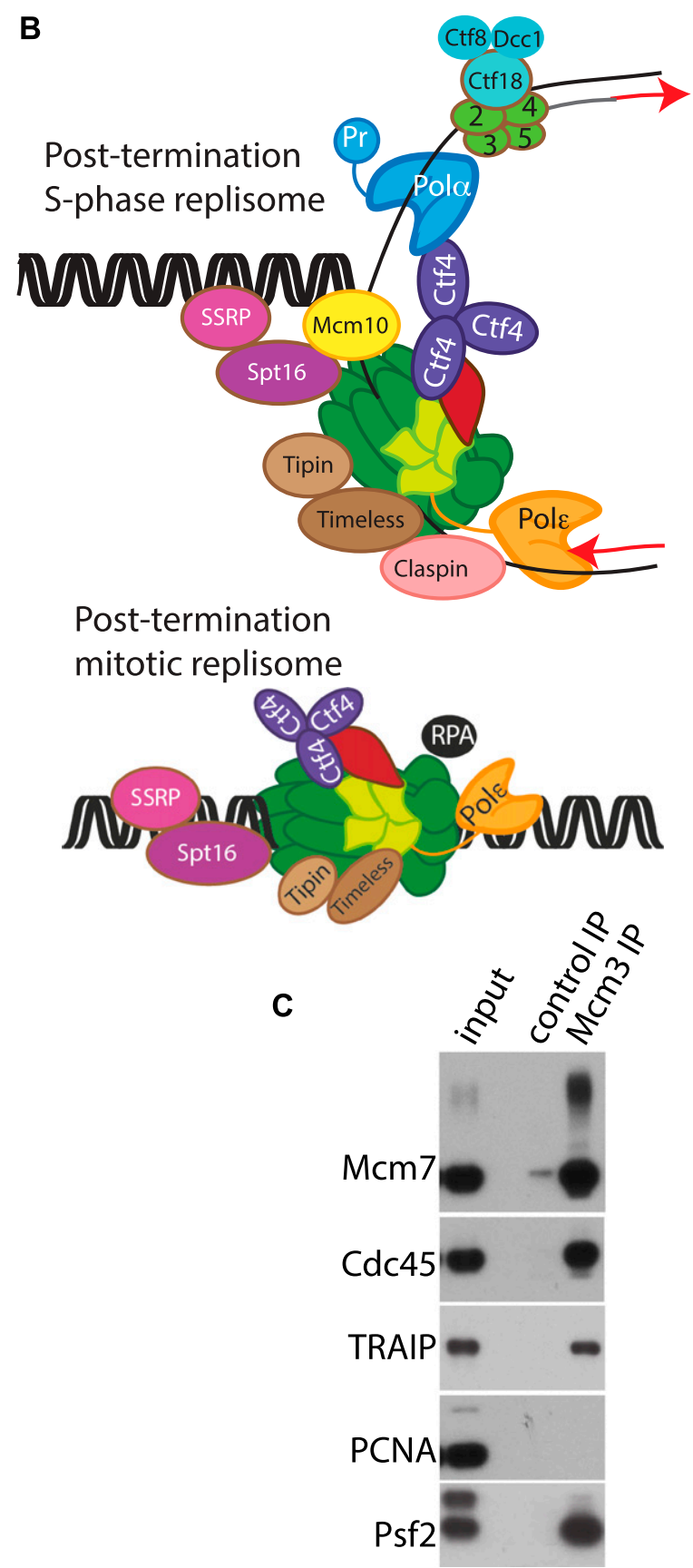

Figure 2. Composition of the replisome retained on mitotic chromatin.

(A) The replication reaction was completed in egg extract supplemented with caffeine and p97 inhibitor NMS873. The extract was then driven into mitosis by addition

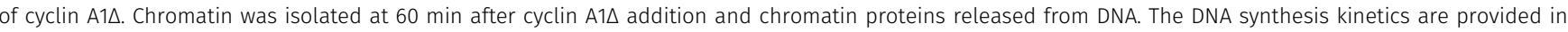
Fig S3A. Antibodies against Mcm3 (or control IgG) were used to immunoprecipitate replisomes, and the immunoprecipitated samples were analysed by mass spectrometry. The total spectral count for each identified replisome component is presented together with sequence coverage of analysed peptides. The results for this analysis of mitotic retained replisome are compared with the S-phase post-replication replisome reported in Sonneville et al (2017). (B) Schematic representation of the data presented in (A). (C) A small proportion of the material from the mitotic Mcm3 IP experiment in (A) was analysed by Western blotting with indicated antibodies.

unloading through ubiquitylation of $\mathrm{Mcm} 7$ in mitosis, we repeated this experiment but supplemented the mitotic extract also with p97 inhibitor NMS873 to inhibit unloading of ubiquitylated Mcm7. Fig 3D shows that addition of enzymatic dead TRAIP, but not wt TRAIP, perturbs mitotic ubiquitylation of $\mathrm{Mcm} 7$ as the ubiquitylated forms of $\mathrm{Mcm} 7$ remain very close in size to the chains built on $\mathrm{Mcm} 7$ already in S-phase (15-min time point). The same prevention of ubiquitylation of Mcm7 in mitosis was observed when we used recombinant GST-tagged TRAIP C25A mutant, but not wt GST-TRAIP (Fig S3F). These results suggest that the recombinant mutant of 
A
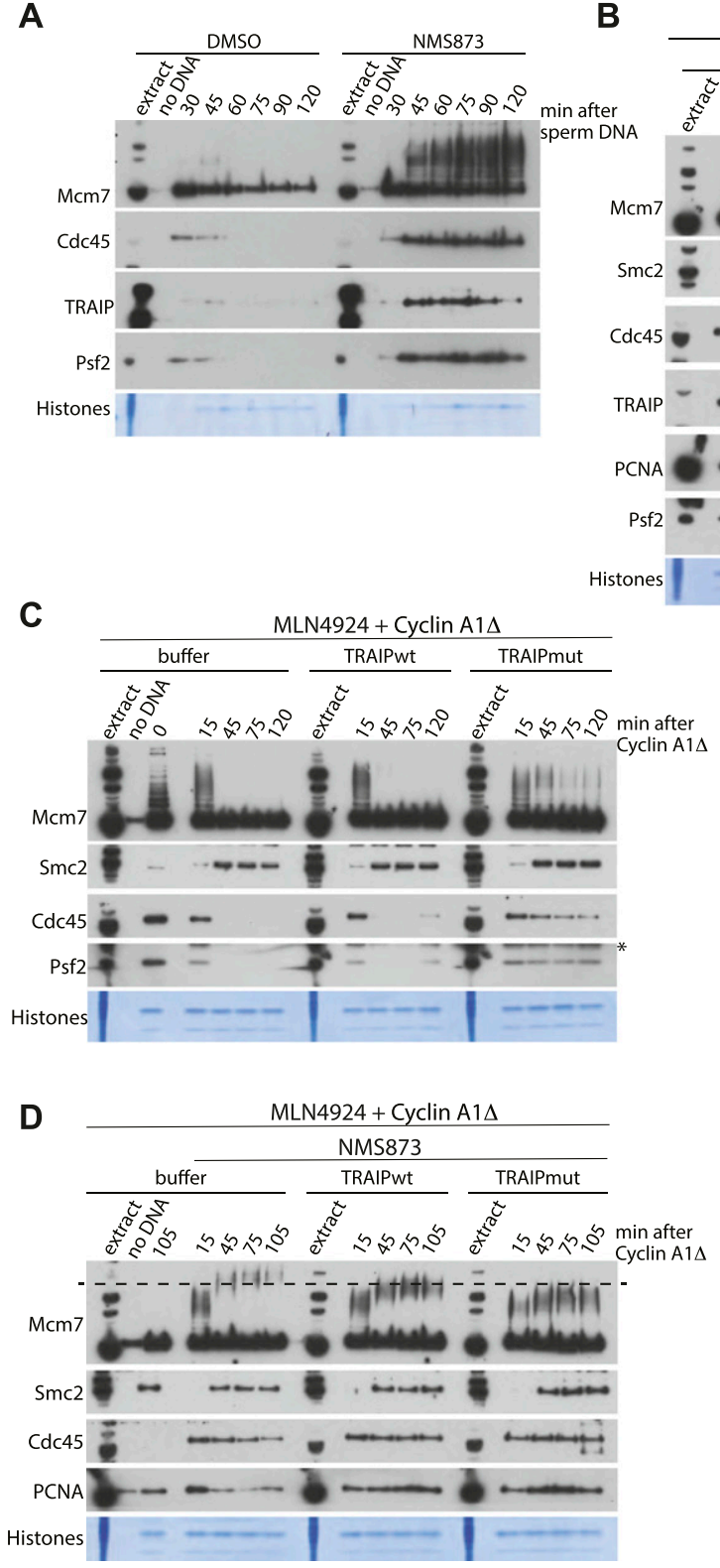
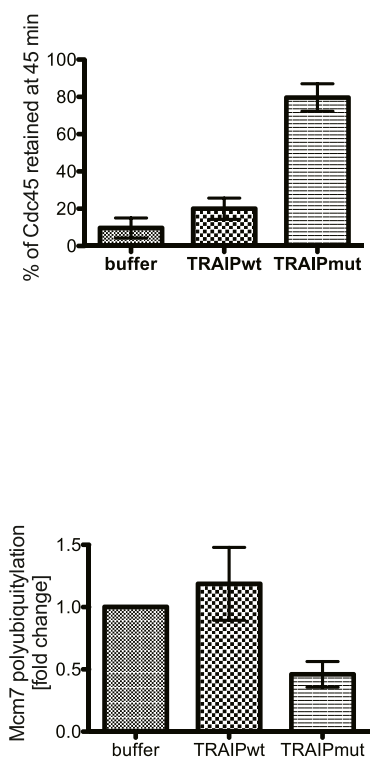

Figure 3. TRAIP ubiquitin ligase drives replisome disassembly in mitosis.

(A) Sperm DNA was replicated in egg extract optionally supplemented with p97 inhibitor NMS873. Chromatin samples were isolated during the reaction at indicated time points and analysed as in Fig 1. Colloidal Coomassie-stained histones serve as a quality and loading control. A sample without DNA addition (no DNA) was processed alongside others as a chromatin specificity control. (B) Experiment analogous to Fig $1 C$ but analysed with indicated antibodies. An asterisk "*" by the Psf2 blot indicates the band of cyclin A1 $\Delta$ that is recognised by the Psf2 antibody. (c) (left) - The replication reaction was completed in the presence of Cullin ligase inhibitor MLN4924 and driven into mitosis by addition of cyclin A1 $\Delta$. At the same time as cyclin A1 $\Delta$, the samples were supplemented optionally with LFB1/ 50 buffer, wt His/SUMO-tagged TRAIP, or RING-mutant (C25A) TRAIP to a final concentration of $50 \mu \mathrm{g} / \mathrm{ml}$. Chromatin samples were isolated at indicated time points and analysed with indicated antibodies. Time " 0 " sample was isolated at the replication completion time when cyclin A1 $\triangle$ and recombinant TRAIP were added to the extract. An asterisk "*" by the Psf2 blot indicates the band of cyclin A1 $\Delta$ that is recognised by the Psf2 antibody. (right)- The level of retained Cdc45 on chromatin was quantified at 15 and $45 \mathrm{~min}$ in each condition and the percentage of the 15 min signal still retained on chromatin at $45 \mathrm{~min}$ calculated. The graph represents a mean of three independent experiments with SEM. (D) (left) - The experiment was performed as in (C) but with addition of p97 inhibitor NMS873 at the same time as cyclin A1 $\Delta$ to block ubiquitylated Mcm7 on chromatin. His/SUMO-tagged TRAIPwt and ligase dead mutant were added to a final concentration of $100 \mu \mathrm{g} /$ $\mathrm{ml}$. The sample isolated at $105 \mathrm{~min}$ without NMS873 provides a control for the unloading without p97 inhibition. The dashed line on the Mcm7 blot runs through the middle of the ubiquitylation signal for $\mathrm{Mcm} 7$ in mitosis in the control (buffer) sample to aid comparison of chain lengths between samples. (right) The Mcm7 polyubiquitylation signal was quantified for each condition as explained in materials and methods and an example provided in Fig S3E. The graph presented here shows the mean fold change of $\mathrm{Mcm} 7$ polyubiquitylation signal at 45 min after cyclin A1 $\triangle$ addition over three independent experiments with SEM.
TRAIP successfully competed with endogenous TRAIP protein and that ubiquitin ligase activity of TRAIP is needed for Mcm7 ubiquitylation and disassembly of post-termination replisome in mitosis. The low level of Mcm7 ubiquitylation visible in samples supplemented with TRAIP mutant is most likely due to the fact that there is still endogenous active TRAIP in the extract.

To fully understand the requirement for ubiquitin-like modifications during mitotic replisome disassembly in vertebrates, we aimed to establish whether SUMOylation plays any role in this process as ULP-4 is essential for mitotic helicase disassembly in C. elegans embryos. To this end, we decided to inhibit or stimulate SuMOylation during mitosis and assess its effect on replisome disassembly. First, we observed that the late S-phase chromatin is full of SUMO2ylated factors and that levels of these proteins go down over time upon entry into mitosis (Fig 4). To inhibit SUMOylation, we supplemented the mitotic extract with the recombinant active domain of SENP1, which acts as a potent nonspecific deSUMOylating enzyme. Addition of SENP1 indeed wiped out all the SUMO2ylation (Fig 4A) and SUM01ylation (Fig S5A), but disassembly of the mitotic replisome is not affected (Fig 4A). We also stimulated SuMOylation through addition of a high concentration of recombinant SUMO1 or SUMO2 (Fig S4). In both cases, despite a clear increase of SUMO signal on chromatin, unloading of the mitotic replisome was not affected. Finally, we also blocked de-SUMOylation with SUMO2-VS, a derivative of SUMO2, which binds to the active site of SENPS and blocks their activity. Again, we observed strong accumulation of SUMO2ylated products in the extract and on chromatin without affecting mitotic replisome disassembly (Fig 4B). Interestingly, despite inhibition of 
A

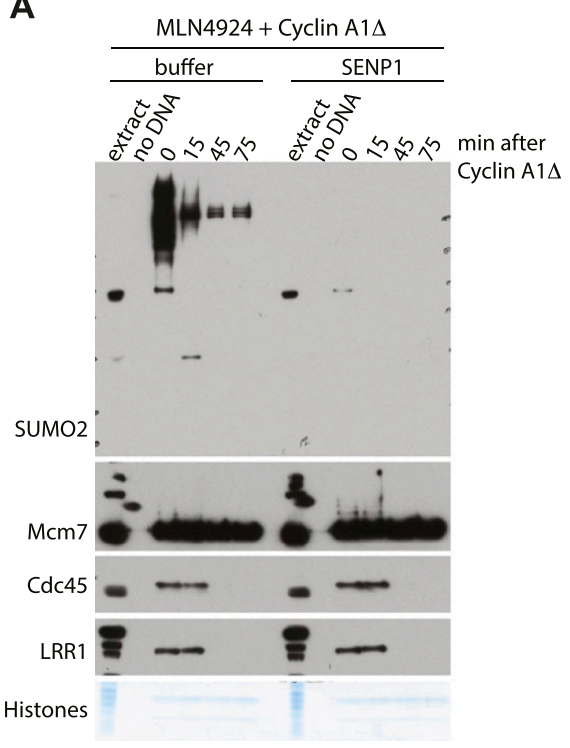

B

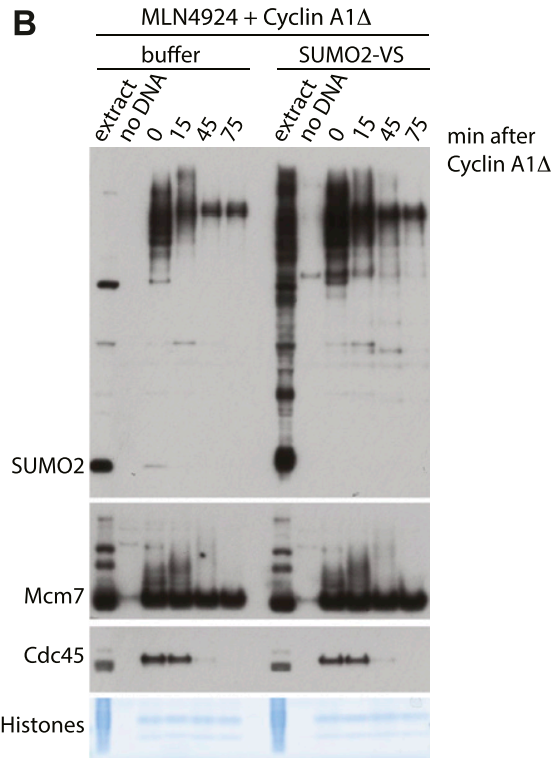

Figure 4. SUMOylation is not required for mitotic replisome disassembly.

(A) The replication reaction was completed in the presence of Cullin ligase inhibitor MLN4924 and driven into mitosis by addition of cyclin A1 $\Delta$. At the same time as cyclin $A 1 \Delta$, half of the sample was supplemented additionally with the active domain of SENP1. Chromatin samples were isolated at indicated time points and analysed as in Fig 1. Colloidal Coomassie-stained histones serve as a quality and loading control. A sample without DNA addition (no DNA) was processed alongside others as a chromatin specificity control. (B) As in (A) but instead of supplementing extract with SENP1, it was supplemented with SENPS inhibitor SUMO2-VS.
de-SuMOylating enzymes, most of the SUMO signal is still disappearing from chromatin during progression of mitosis, indicating that the SUMOylated proteins are unloaded from chromatin throughout mitosis rather than being de-SUMOylated. In conclusion, we determined that SUMO modifications do not play an essential role in the mitotic replisome disassembly pathway in Xenopus egg extract. In an analogous way, we have also shown that they do not play a role during the $\mathrm{S}$-phase replisome disassembly pathway (Fig S5A and B).

Finally, we set out to determine whether this mitotic replisome disassembly pathway was a mere "backup" pathway for replisomes that terminated in S-phase but failed to be unloaded, or if it has a more generic ability to remove any replication machinery still remaining on chromatin in mitosis. To test such a possibility, we stalled replisomes on chromatin by addition of DNA polymerase inhibitor aphidicolin to the egg extract during the DNA replication reaction. To accumulate such replisomes in large numbers, we also supplemented the extract with caffeine so as to block checkpoint activation and fire origins uncontrollably. Upon accumulation of such blocked replisomes, we supplemented the reaction optionally with cyclin A1 $\triangle$ at 90 min to induce mitotic entry (Fig 5A). Addition of cyclin A1 $\triangle$ did not stimulate any more DNA synthesis in our samples (Fig S6). Interestingly, active replisomes remained associated with chromatin throughout the experiment in late S-phase (buffer), with no indication of Mcm7 ubiquitylation as expected (Moreno et al, 2014). Upon addition of cyclin A1 $\Delta$, however, Mcm7 became ubiquitylated and replisomes were unloaded (Fig 5A, cyclin A1 4 ), although we did observe a slight delay in both of these processes compared with terminated replisomes (compare Fig 5A with Fig 1B). Such a delay is likely due to the fact that with no prior ubiquitylation of Mcm7 in S-phase, it takes longer for ubiquitin chains to be built in mitosis. We also determined that unloading of stalled replisomes requires the activity of $\mathrm{p} 97$ segregase, as unloading is inhibited in the presence of the p97 inhibitor NMS873 (Fig 5B). Finally, to test whether the activity of TRAIP ubiquitin ligase is needed for the unloading of stalled replisomes, we added recombinant wt or enzymatic dead TRAIP to mitotic extract and observed its effect on unloading of such stalled replisomes. As with post-termination replisomes (Fig $3 C$ and D), the enzymatic dead mutant of TRAIP inhibited unloading of stalled helicase (Fig 5C), whereas we also observed a clear reduction in the ubiquitylation of stalled $\mathrm{Mcm} 7$ (Fig 5D). From these observations, we can thus say that neither prior modification of Mcm7 in S-phase nor the "terminated" conformation of the helicase are essential for mitotic modification of $\mathrm{Mcm} 7$ by TRAIP and subsequent replisome disassembly.

\section{Discussion}

We have presented here the existence of a mitotic pathway of replisome disassembly in $X$. laevis egg extract. One immediate question is why would the cells need a mitotic pathway of replisome disassembly? Traditionally, it is perceived that all DNA metabolism should be finished before cells enter mitosis. According to this model, the G2 phase of the cell cycle is there to ensure that all DNA replication and damage repair are completed before chromosome condensation and segregation during mitosis. The last decade provided, however, much evidence that this is not the case: unreplicated DNA is detected in many human cells in mitosis; DNA synthesis can proceed during mitosis (mitotic DNA synthesis-MiDAS); under-replicated DNA can lead to the formation of ultrafine bridges in anaphase and structures in the G1 stage of the next cell cycle that are bound by 53BP1 protein (53BP1 bodies) (Liu et al, 2014; Minocherhomji et al, 2015; Moreno et al, 2016). Genome-wide, such unreplicated regions correlate with common fragile sites, which are chromosomal loci responsible for the majority of the rearrangements found in cancer cells (Bhowmick \& Hickson, 2017). These unreplicated fragments of DNA result from replication forks not finishing replication and such forks, with their associated replisomes, 
A
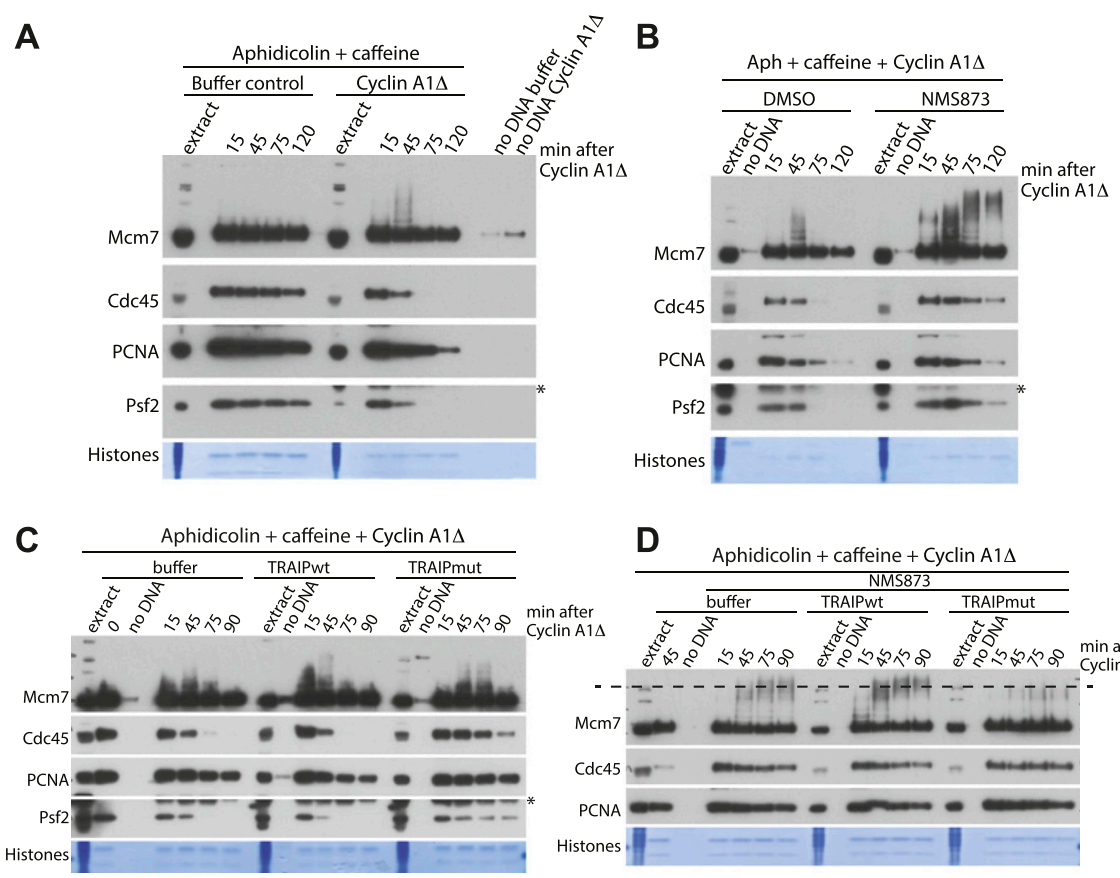

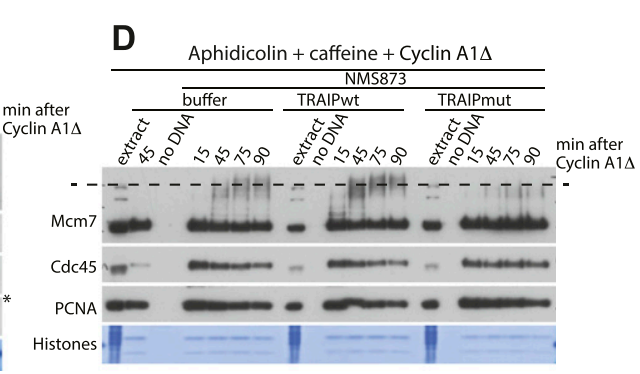

Figure 5. Mitotic unloading of stalled helicases. (A) The replication reaction was performed in egg extract supplemented with DNA polymerase inhibitor aphidicolin and checkpoint inhibitor caffeine. After 90 min of reaction, cyclin $\mathrm{A} 1 \Delta$ was optionally added, and the chromatin samples were isolated during the reaction at indicated time points and analysed as in Fig 1. Colloidal Coomassie-stained histones serve as a quality and loading control. A sample without DNA addition (no DNA) was processed alongside others as a chromatin specificity control. An asterisk "*" by the Psf2 blot indicates the band of cyclin A1 $\triangle$ that is recognised by the Psf2 antibody. (B) The inhibition of stalled replisomes was achieved as in (A), and the extract was driven into mitosis by addition of cyclin A1 $\triangle$ and optional supplementation with p97 inhibitor NMS873. The chromatin samples were analysed as in (A). (C) Replication reaction was performed with addition of aphidicolin and caffeine, at $90 \mathrm{~min}$, cyclin A1 $\triangle$ was added and optionally: buffer, His/SUMO-TRAIPwt, or ligase dead His/SUMO-TRAIPmut (C25A) to a final concentration of $50 \mu \mathrm{g} / \mathrm{ml}$. The chromatin samples were isolated at indicated time points and analysed with indicated antibodies. Time "0" sample was isolated at the replication completion time when cyclin $A 1 \Delta$ and recombinant TRAIP were added to the extract. (D) The experiment was performed as in (C) but with addition of p97 inhibitor NMS873 at the same time as cyclin A1 $\Delta$ to accumulate ubiquitylated forms of $\mathrm{Mcm} 7$ on chromatin. His/SUMO-tagged TRAIPwt and mutant were added to a final concentration of $100 \mu \mathrm{g} / \mathrm{ml}$. The sample isolated at 45 min without NMS873 provides a control for the replisome unloading without p97 inhibition. The dashed line on the Mcm7 blot runs through the middle of the ubiquitylation signal for Mcm7 in mitosis in the control (buffer) sample to aid comparison of chain lengths between samples.

are subsequently retained on chromatin into mitosis. It is likely that these unreplicated DNA fragments must be processed in mitosis to ensure correct chromosome segregation, and this processing will involve replisome unloading and fork remodelling -hence the need for a process of replisome disassembly in mitosis.

TRAIP is a pleiotropic ubiquitin ligase involved in numerous cellular processes. It is clear that TRAIP is essential for appropriate repair of DNA damage in many forms: mitomycin C-induced interstrand crosslinks; damage caused by treatments with camptothecin (Hoffmann et al, 2016), UV (Harley et al, 2016) and hydroxyurea (Feng et al, 2016); as well as for translesion DNA synthesis (Wallace et al, 2014). TRAIP has also been reported to be an important regulator of the spindle assembly checkpoint and regulates mitotic progression (Chapard et al, 2014; Park et al, 2015). For most of these processes, the ubiquitin ligase activity of TRAIP is essential, but the substrate (s) modified by TRAIP is not known.

In support of our observation that TRAIP interacts weakly with the S-phase chromatin when replication forks replicate DNA (Fig $3 A$ ), TRAIP has been shown to interact with nascent DNA in unperturbed S-phase in human cells through nascent chromatin capture (Hoffmann et al, 2016), but TRAIP knockdown does not significantly affect replication progression and overall DNA synthesis rates (Harley et al, 2016; Hoffmann et al, 2016). Upon DNA damage, TRAIP relocalises from nucleoli to sites of damage in a manner dependent on a PCNA interacting box (PIP-box), present at the $C$ terminus of TRAIP (Feng et al, 2016; Hoffmann et al, 2016). Loss of TRAIP was suggested to interfere with the reconfiguration of stalled replication forks (possibly through unloading of PCNA) (Hoffmann et al, 2016), as further inhibition of proteasomal degradation in the absence of TRAIP did not exacerbate the levels of hydroxyurea- induced fork stalling. This suggested that degradation of a TRAIP ubiquitylation substrate is not the cause of this phenotype (Feng et al, 2016; Hoffmann et al, 2016). Interestingly, cells expressing the $\triangle$ RING mutant of TRAIP as the only TRAIP version, are as sensitive to mitomycin $\mathrm{C}$ as TRAIP knockdown cells, while $\triangle$ PIP TRAIP cells are only mildly sensitive. This indicates that even without PCNA interaction, TRAIP can still find its targets at the replication forks (Hoffmann et al, 2016). With the data presented here, identifying TRAIP as the ubiquitin ligase needed for Mcm7 ubiquitylation during mitosis, it is interesting to speculate that TRAIP can play an analogous role during DNA damage repair, that is, to stimulate replisome unloading and fork remodeling. Indeed, recently TRAIP has been shown to ubiquitylate CMG during inter-strand crosslink (ICL) repair for replisome unloading (Wu et al, 2019).

Our data are consistent with a model in which TRAIP drives mitotic replisome disassembly by promoting $\mathrm{Mcm} 7$ modification with $\mathrm{K} 6$ and K63-linked ubiquitin chains. Although there is no previous experimental evidence that TRAIP can support such ubiquitin linkages in vivo, in vitro assays have shown that TRAIP works well with conjugating enzymes (E2s) UbcH5a,b, and c (but not $\mathrm{UbcH} 2, \mathrm{H} 3, \mathrm{H} 6, \mathrm{H} 7$, or Ubc13+Uev1A) (Besse et al, 2007) and (Fig S3C). Interestingly, UbcH5a was shown to support formation of ubiquitin chains with no specific topology (Windheim et al, 2008). It is, therefore, plausible that TRAIP/UbcH5 can effectively produce chains of different linkages to support mitotic replisome disassembly.

Replisome disassembly in S-phase is driven by $\mathrm{Mcm} 7$ ubiquitylation, specifically with K48-linked ubiquitin chains. In mitosis, however, K48-linked chains are not functional and unloading is driven instead by K6- and K63-linked chains. We know that p97, in complex with Ufd1 and Npl4 cofactors, is responsible for 
unloading of the replisome in S-phase (Moreno et al, 2014; Maric et al, 2017; Sonneville et al, 2017). Although p97 is well known for processing substrates ubiquitylated with K48-linked ubiquitin chains (Meyer et al, 2012), less is known about its contribution in processing other ubiquitin linkages. Interestingly, a recent study shows that upon inhibition of p97 activity, human cells accumulate K6-, K11-, K48- and, to a lesser extent, K63-linked ubiquitin chains (Heidelberger et al, 2018). Moreover, out of five tested p97 cofactors, all were found to associate with K11 chains, four with K48 chains, and three with $\mathrm{K} 63$ chains (Alexandru et al, 2008). p97 cofactors are known also to interact with ubiquitin-like modifiers, for example, Nedd8 and Atg8 (reviewed in Meyer (2012)). Finally, p97 was also shown to bind more readily to branched K11-K48 chains than to K11 or K48 chains on their own (Meyer \& Rape, 2014). These proteomewide data imply that the role of p97 does indeed extend beyond recognition of K48-chain-modified substrates, although currently, little is known about its interaction with $\mathrm{K} 6$ chains.

Finally, we have shown that in the Xenopus system, neither the S-phase (Fig S5) nor the mitotic replisome disassembly requires SUMO modifications (Figs 4 and S4) in contrast to C. elegans embryos where ULP-4 is required for mitotic unloading (Sonneville et al, 2017). This requirement may be specific to worm embryos, require ULP-4 protein but not its enzymatic activity, or it may regulate an indirect process that is not well recapitulated in the egg extract cell-free system. Of note, it has been suggested recently that SUMOylation of TRAIP can regulate its stability and ability to move to the nucleus (Park et al, 2016), but this may not be present in the egg extract.

Perturbations in DNA replication initiation and elongation leading to genomic instability are well linked with genetic disorders and can drive cancer development. The disruption of replisome disassembly is, therefore, highly likely to be detrimental to human health too. Although so far we have no solid data to support this claim, previous studies with TRAIP do suggest this to be the case: homozygous TRAIP knockout mouse embryos die shortly after implantation because of proliferation defects (Park et al, 2007); mutations in human TRAIP lead to primordial dwarfism (Harley et al, 2016); overexpression of human TRAIP has been reported in basal cell carcinomas (Almeida et al, 2011) and breast cancer (Yang et al, 2006; Zhou \& Geahlen, 2009); and reduced nuclear expression of TRAIP was associated with human lung adenocarcinoma (Soo Lee et al, 2016). The fact that cells have evolved multiple pathways to ensure timely replisome disassembly supports the notion of the vital importance of this process for cell biology, and time will tell whether targeting Mcm7 and replisome disassembly in mitosis is the key mechanism leading to any of these disease phenotypes.

\section{Materials and Methods}

\section{Inhibitors}

Caffeine (C8960; Sigma-Aldrich) was dissolved in water at $100 \mathrm{mM}$ and added to the extract along with demembranated sperm nuclei at 5 mM. MLN4924 (A01139; Active Biochem) was dissolved in DMSO at $20 \mathrm{mM}$ and added to the extract $15 \mathrm{~min}$ after addition of sperm nuclei at $10 \mu \mathrm{M}$. NMS873 (17674; Cayman Chemical Company) was dissolved in DMSO at $10 \mathrm{mM}$ and added to the extract 15 min after addition of sperm nuclei at $50 \mu \mathrm{M}$. SUMO2-VS (UL-759) was purchased from Boston Biochem and used at $1 \mu \mathrm{M}$ in X. laevis egg extract. Aphidicolin was dissolved in DMSO at $8 \mathrm{mM}$ and added to the extract along with demembranated sperm nuclei at $40 \mu \mathrm{M}$.

\section{Recombinant proteins}

Recombinant His-tagged ubiquitin and ubiquitin mutants were purchased from Boston Biochem, dissolved in LFB1/50 $(40 \mathrm{mM}$ Hepes/ $\mathrm{KOH}, \mathrm{pH} 8.0,20 \mathrm{mM}$ potassium phosphate, $\mathrm{pH} 8.0,50 \mathrm{mM} \mathrm{KCl}$, $2 \mathrm{mM} \mathrm{MgCl} 2,1 \mathrm{mM}$ EGTA, $10 \%$ sucrose wt/vol, $2 \mathrm{mM} \mathrm{DTT}, 1 \mu \mathrm{g} / \mathrm{ml}$ aprotinin, $1 \mu \mathrm{g} / \mathrm{ml}$ leupeptin, and $1 \mu \mathrm{g} / \mathrm{ml}$ pepstatin) buffer at 10 $\mathrm{mg} / \mathrm{ml}$, and used at $0.5 \mathrm{mg} / \mathrm{ml}$ in $X$. laevis egg extract.

pET28a-X.L.SUMO1 and pET28a-X.L.SUMO2 were purchased from GenScript. Recombinant His-tagged X. laevis SUMO1 and SUMO2 were expressed in Rosetta (DE3) pLysS cells over night at $20^{\circ} \mathrm{C}$ after induction with $1 \mathrm{mM} \mathrm{IPTG}$. The cells were lysed in lysis buffer: $50 \mathrm{mM}$ Tris- $\mathrm{HCl}, 500 \mathrm{mM} \mathrm{NaCl}, 10 \mathrm{mM}$ imidazole, $2 \mathrm{mM} \mathrm{MgCl}_{2}$, $0.1 \mathrm{mM}$ PMSF, and $1 \mu \mathrm{g} / \mathrm{ml}$ of each aprotinin, leupeptin, and pepstatin, $\mathrm{pH} 7.5$. Homogenates were supplemented with $25 \mathrm{U} / \mathrm{ml}$ benzonase and incubated at room temperature for $20 \mathrm{~min}$. Homogenates were subsequently spun down at $14,000 \mathrm{~g}$ for $30 \mathrm{~min}$ at $4^{\circ} \mathrm{C}$ and supernatants incubated with $2 \mathrm{ml}$ of prewashed Super Ni-NTA Affinity Resin (SUPER-NINA100; Generon) for $2 \mathrm{~h}$ with rotation at $4^{\circ} \mathrm{C}$. Resins were subsequently washed twice with $50 \mathrm{mM} \mathrm{Tris}-\mathrm{HCl}$, $500 \mathrm{mM} \mathrm{NaCl}, 30 \mathrm{mM}$ imidazole, $0.1 \mathrm{mM}$ PMSF, and $1 \mu \mathrm{g} / \mathrm{ml}$ of each aprotinin, leupeptin, and pepstatin, $\mathrm{pH}$ 7.5. Resin-bound proteins were finally eluted in $1 \mathrm{ml}$ fractions with a solution containing 50 $\mathrm{mM}$ Tris- $\mathrm{HCl}, 150 \mathrm{mM} \mathrm{NaCl}, 200 \mathrm{mM}$ imidazole, $5 \mathrm{mM} \beta$ mercaptoethanol, $0.1 \mathrm{mM}$ PMSF, and $1 \mu \mathrm{g} / \mathrm{ml}$ of each aprotinin, leupeptin, and pepstatin, $\mathrm{pH}$ 7.5. Fractions containing the highest levels of recombinant SUMO1 or SUMO2 were dialysed into LFB1/50 buffer. Both SUMO1 and SUMO2 were used at $0.5 \mathrm{mg} / \mathrm{ml}$ in X. laevis egg extract.

pET28a-pHISTEV30a-SENP1(415-649) was a kind gift from Prof Ron Hay's laboratory. Recombinant active domain of human SENP1 (aa 415-647) was expressed and purified as explained above for recombinant SUMOs.

Recombinant His-tagged $X$. laevis cyclin A1 N $\Delta 56$ (pET23a-X.l. Cyclin A1 N $\triangle 56$ ) was a kind gift from Prof Julian Blow's laboratory (Strausfeld et al, 1996), was expressed in Rosetta (DE3) pLysS cells over night at $15^{\circ} \mathrm{C}$ after induction with $1 \mathrm{mM} \mathrm{IPTG}$, and subsequently purified as explained above for recombinant SUMOs but using different solutions. Lysis buffer: $50 \mathrm{mM}$ Tris-HCl, $300 \mathrm{mM} \mathrm{NaCl}, 2 \mathrm{mM}$ $\mathrm{MgCl}_{2}, 1 \mathrm{mM}$ DTT, $0.1 \mathrm{mM}$ PMSF, and $1 \mu \mathrm{g} / \mathrm{ml}$ of each aprotinin, leupeptin, and pepstatin, $\mathrm{pH}$ 7.4. Washes: Resin was washed twice with lysis buffer on its own and twice again with lysis buffer supplemented with $0.1 \%$ Triton X-100. Elution buffer: Lysis buffer supplemented with $10 \%$ glycerol and $250 \mathrm{mM}$ imidazole.

Xenopus TRAIP was cloned into pGS21 vector, expressed in BL21 (DE3) bacterial strain in Auto Induction Media (AIM) media (Formedium) $\mathrm{O} / \mathrm{N}$ at $18^{\circ} \mathrm{C}$. Pellets were lysed in lysis buffer: $50 \mathrm{mM}$ $\mathrm{NaH}_{2} \mathrm{PO}_{4}, \mathrm{pH}$ 9; $300 \mathrm{mM} \mathrm{NaCl} ; 10 \%$ glycerol; $2 \mathrm{mM} \mathrm{DTT} ; 2 \mathrm{mM} \mathrm{MgCl}$; 0.05\% Brij; $0.1 \mathrm{mM}$ PMSF; $1 \mu \mathrm{g} / \mathrm{ml}$ of each aprotinin, leupeptin, and pepstatin; $1 \mathrm{mg} / \mathrm{ml}$ lysozyme; and $25 \mathrm{U} / \mathrm{ml}$ benzonase. The protein was purified as above but using Glutathione Sepharose 4B (GE 
Healthcare) and eluted with $25 \mathrm{mM}$ glutathione. The protein was then dialysed into LFB1/50 buffer (as above) and concentrated up to $0.3 \mathrm{mg} / \mathrm{ml}$ of full-length GST-TRAIP. It was used in the egg extract at a final concentration of $30 \mu \mathrm{g} / \mathrm{ml}$. pGS21-TRAIP(C25A) was generated by site-directed mutagenesis and purified in an analogous way.

Recombinant His/SUMO-tagged $X$. laevis wt and mutant TRAIP were expressed in Rosetta (DE3) pLys $S$ cells from $\mathrm{pCA} 528$ vector $\mathrm{O} / \mathrm{N}$ at $20^{\circ} \mathrm{C}$ in AIM media. After pelleting of the bacterial cultures, the cells were lysed in $50 \mathrm{mM} \mathrm{NaH}_{2} \mathrm{PO}_{4}, 500 \mathrm{mM} \mathrm{NaCl}, 0.05 \%$ Brij, $10 \%$ glycerol, $10 \mathrm{mM}$ imidazole, $2 \mathrm{mM} \mathrm{MgCl} 2,0.1 \mathrm{mM} \mathrm{PMSF}$, and $1 \mu \mathrm{g} / \mathrm{ml}$ of each aprotinin, leupeptin, and pepstatin, $\mathrm{pH}$ 9.0. Homogenates were supplemented with $1 \mathrm{mg} / \mathrm{ml}$ lysozyme and $25 \mathrm{U} / \mathrm{ml}$ BaseMuncher, incubated at room temperature for $20 \mathrm{~min}$ and subsequently sonicated $(6 \times 30 \mathrm{~s})$, and spun $\left(30 \mathrm{~min}, 31,000 \mathrm{~g}, 4^{\circ} \mathrm{C}\right)$. The resulting supernatant was incubated in $2 \mathrm{ml}$ prewashed Super Ni-NTA Affinity Resin (SUPER-NINA100; Generon) O/N with rotation at $4^{\circ} \mathrm{C}$. Resins were washed five times with lysis buffer, with respect to the following alterations: Wash 1: $100 \mathrm{mM} \mathrm{NaCl}$, no imidazole. Wash 2: 100 mM NaCl, 20 mM imidazole. Wash 3: $250 \mathrm{mM} \mathrm{NaCl}$, no imidazole. Wash 4 and 5: $500 \mathrm{mM} \mathrm{NaCl}, 20 \mathrm{mM}$ imidazole. Each respective wash was supplemented with $0.1 \mathrm{mM}$ PMSF and $1 \mu \mathrm{g} / \mathrm{ml}$ of each aprotinin, leupeptin, and pepstatin. Resin-bound TRAIP was eluted in 1-ml fractions using elution buffer $\left(50 \mathrm{mM} \mathrm{NaH}_{2} \mathrm{PO}_{4}, 500 \mathrm{mM} \mathrm{NaCl}, 0.05 \%\right.$ Brij, 10\% Glycerol, 400 mM imidazole, pH 9.0) supplemented with 0.1 $\mathrm{mM}$ PMSF and $1 \mu \mathrm{g} / \mathrm{ml}$ of each aprotinin, leupeptin, and pepstatin. Those fractions containing the highest quantities of wt or mutant TRAIP were dialysed into LFB1/100 buffer.

\section{In vitro TRAIP autoubiquitylation reaction}

The reaction was set up as previously described (Besse et al, 2007). Briefly, GST-TRAIPwt and GST-TRAIPmut were purified as described above but not eluted from Glutathione Sepharose beads. Both beads were then incubated in $100-\mu \mathrm{l}$ reaction for $2 \mathrm{~h}$ at $37^{\circ} \mathrm{C}$ with 20 mM Hepes, pH 7.4, 10 mM MgCl 2,1 mM DTT, $60 \mu \mathrm{M}$ His-Ubi (Boston Biotech), 50 nM E1 (UBA1/UBE1; Source BioScience), 850, nM E2 UbcH5a (Source BioScience), $1 \mathrm{mM}$ ATP, $30 \mu \mathrm{M}$ creatine phosphate, and $1 \mathrm{U}$ of creatine kinase. After incubation, they were extensively washed, boiled in gel-loading buffer, and run on the gel. The membrane was analysed with $\alpha$-ubiquitin antibody.

\section{Antibodies}

$\alpha$-PCNA (P8825), $\alpha$-His (H1029), and $\alpha$-ubiquitin (P4D1) were purchased from Sigma-Aldrich and $\alpha$-phospho-histone H3 (ser10) (D2C8) was purchased from Cell Signalling Technology. $\alpha$-TRAIP (NBP1-87125) and $\alpha$-RNF213 (NBP1-88466) were purchased from Novus Biologicals. $\alpha$-SUMO2 and $\alpha$-SUMO1 were produced in the laboratory by culturing the hybridoma cell lines SUMO2 (8A2), and SUMO1 (21C7), purchased from Developmental Studies Hybridoma Bank (hybridoma cell culture was performed following the manufacturer's instructions and adding $20 \mathrm{mM} \mathrm{L-glutamine} \mathrm{to} \mathrm{the} \mathrm{media).} \mathrm{Affinity-purified}$ $\alpha$-Cdc45, $\alpha$-Psf2, and $\alpha$-SId5 (Gambus et al, 2011); $\alpha$-Mcm3 (Khoudoli et al, 2008); $\alpha$-SMC2 (Gillespie et al, 2007); and $\alpha$-LRR1 (S962D) (Sonneville et al, 2017) were previously described. $\alpha$-Mcm7 was raised in sheep against recombinant $X$. laevis $M c m 7$, purified from E. coli, and affinity-purified in the laboratory.

\section{DNA staining and microscopy}

Interphase $X$. laevis egg extract was supplemented with $10 \mathrm{ng} / \mu \mathrm{l}$ of demembranated sperm nuclei and incubated at $23^{\circ} \mathrm{C}$ until completion of DNA replication as described before (Gillespie et al, 2012). Mitosis was optionally driven by addition of $826 \mathrm{nM}$ cyclin A1 N $\Delta 56$. At time points $-30,0,30$, and 60 min, after addition of cyclin $A 1,10 \mu \mathrm{l}$ of the reaction was spotted onto a microscope slide with $10 \mu \mathrm{l}$ mix of Hoechst 33258 (5824/50; Tocris Bioscience) and Dil stain (D282; Thermo Fisher Scientific), $1 \mu \mathrm{g} / \mathrm{ml}$ and $10 \mu \mathrm{g} / \mathrm{ml}$ final concentration, respectively, and incubated at room temperature for $30 \mathrm{~min}$. Nuclei were viewed as previously described (Strausfeld et al, 1996).

\section{DNA synthesis assay}

The replication reactions were started with the addition of demembranated Xenopus sperm DNA to $10 \mathrm{ng} / \mu \mathrm{l}$ as described before (Gillespie et al, 2012). The synthesis of nascent DNA was measured by quantification of $\mathrm{P}^{32} \alpha$-dATP incorporation into newly synthesised DNA as described before (Gillespie et al, 2012).

\section{Chromatin isolation time-course}

Interphase $X$. laevis egg extract was supplemented with $10 \mathrm{ng} / \mu \mathrm{l}$ of demembranated sperm DNA and subjected to indicated treatments. The reaction was incubated at $23^{\circ} \mathrm{C}$ for $90 \mathrm{~min}$ to allow completion of DNA replication as described before (Gillespie et al, 2012), after which mitosis was optionally driven by addition of 826 $\mathrm{nM}$ cyclin A1 N $\Delta 56$. The extract was then also optionally supplemented with inhibitors or recombinant proteins as indicated. Chromatin was isolated in ANIB100 buffer supplemented with 10 $\mathrm{mM}$ 2-chloroacetamide (Millipore) and $5 \mathrm{mM} \mathrm{N}$-ethylmaleimide (Acros Organics) at indicated time points after addition of cyclin A1 N $\Delta 56$ as previously described (Gillespie et al, 2012).

For the minimal licensing experiment (Fig S2A), interphase $X$. laevis egg extract was supplemented with $5 \mathrm{ng} / \mu \mathrm{l}$ of demembranated sperm DNA. To minimally license chromatin, Cdt1 activity was blocked through addition of geminin ${ }^{\text {DEL }}$ (Blow lab) after 2 min of sperm DNA addition. The extract was optionally supplemented with DMSO and Cullin ligase inhibitor (MLN4924 at $10 \mu \mathrm{M}$ ).

\section{Quantification of Western blots}

Western blot films from three independent experiments were scanned to generate high-resolution, $300 \mathrm{dpi}$, 8-bit JPEG images. The pixel intensity of protein bands was then quantified with Image J (http://rsbweb.nih.gov/ij/) and the average intensity (a.u.) calculated for each time point. For quantification of Mcm7 ubiquitylation, a frame was first drawn around the entire ubiquitylation signal to include that which was built in S-phase and that which was extended further in mitosis. This generates a plot for each sample. To then measure only the intensity of ubiquitylation which occurs in mitosis, a line was drawn through the plots to separate the lower region (S-phase) and the upper region (mitosis). The intensity of 
this upper region was then measured and a.u. calculated from three independent experiments.

\section{Immunoprecipitation of post-termination CMG associated with mitotic chromatin}

$3.75 \mathrm{ml}$ of interphase $X$. laevis egg extract was supplemented with 10 $\mathrm{ng} / \mu \mathrm{l}$ of demembranated sperm nuclei, $5 \mathrm{mM}$ caffeine, and $50 \mu \mathrm{M}$ p97 inhibitor NMS873. The reaction was incubated at $23^{\circ} \mathrm{C}$ for $90 \mathrm{~min}$ to allow completion of DNA replication, after which mitosis was driven by addition of recombinant cyclin $\mathrm{A} 1 \mathrm{~N} \Delta 56$ at $826 \mathrm{nM}$ followed by incubation at $23^{\circ} \mathrm{C}$ for a further $60 \mathrm{~min}$. At this stage, chromatin was isolated as described above and chromatin-bound protein complexes released into solution by chromosomal DNA digestion with 2 $\mathrm{U} / \mu \mathrm{l}$ benzonase for $15 \mathrm{~min}$. Solubilisation of chromatin-bound protein complexes was further facilitated by subjecting the sample to 5 min of $30 \mathrm{~s}$ ON/OFF sonication cycles using a diagenode bioruptor and increasing the concentration of potassium acetate up to $150 \mathrm{mM}$. The resulting protein complexes were subsequently subjected to either nonspecific IgG (from sheep serum) or Mcm3 immunoprecipitation and the immunoprecipitated material analysed by mass spectrometry as previously described (Sonneville et al, 2017) in collaboration with Dr Richard Jones from MS Bioworks LLC.

\section{Supplementary Information}

Supplementary Information is available at https://doi.org/10.26508/lsa. 201900390.

\section{Acknowledgements}

Dr S Priego Moreno was funded by Wellcome Trust Institutional Strategic Support Fund (ISSF) Award, Dr RM Jones and Dr A Gambus were funded by MRC CDA MR/K007106/1 and Shaun Scaramuzza by Midlands Integrative Biosciences Training Partnership (MIBTP) studentship. We would like to thank Prof Ron Hay for pET28a-pHISTEV30a-SENP1(415-649) and Prof Julian Blow for pET23a-X.L.Cyclin A1 N $\triangle 56$ and geminin ${ }^{\text {DEL. }}$

\section{Author Contributions}

S Priego Moreno: conceptualization, data curation, investigation, and writing-review and editing.

RM Jones: data curation, investigation, methodology, and writingreview and editing.

D Poovathumkadavil: data curation, investigation, and writingreview and editing.

S Scaramuzza: data curation and writing-review and editing.

A Gambus: conceptualization, formal analysis, supervision, funding acquisition, project administration, and writing-original draft, review, and editing.

\section{Conflict of Interest Statement}

The authors declare that they have no conflict of interest.

\section{References}

Alexandru G, Graumann J, Smith GT, Kolawa NJ, Fang R, Deshaies RJ (2008) UBXD7 binds multiple ubiquitin ligases and implicates p97 in HIF1alpha turnover. Cell 134: 804-816. doi:10.1016/j.cell.2008.06.048

Almeida S, Ryser S, Obarzanek-Fojt M, Hohl D, Huber M (2011) The TRAFinteracting protein (TRIP) is a regulator of keratinocyte proliferation. J Invest Dermatol 131: 349-357. doi:10.1038/jid.2010.329

Banh RS, Iorio C, Marcotte R, Xu Y, Cojocari D, Rahman AA, Pawling J, Zhang W, Sinha A, Rose CM, et al (2016) PTP1B controls non-mitochondrial oxygen consumption by regulating RNF213 to promote tumour survival during hypoxia. Nat Cell Biol 18: 803-813. doi:10.1038/ncb3376

Besse A, Campos AD, Webster WK, Darnay BG (2007) TRAF-interacting protein (TRIP) is a RING-dependent ubiquitin ligase. Biochem Biophys Res Commun 359: 660-664. doi:10.1016/j.bbrc.2007.05.149

Bhowmick R, Hickson ID (2017) The "enemies within": Regions of the genome that are inherently difficult to replicate. F1000Res 6: 666. doi:10.12688/ f1000research.11024.1

Burrell RA, McClelland SE, Endesfelder D, Groth P, Weller MC, Shaikh N, Domingo E, Kanu N, Dewhurst SM, Gronroos E, et al (2013) Replication stress links structural and numerical cancer chromosomal instability. Nature 494: 492-496. doi:10.1038/nature11935

Chapard C, Hohl D, Huber M (2015) The TRAF-interacting protein (TRAIP) is a novel E2F target with peak expression in mitosis. Oncotarget 6: 20933-20945. doi:10.18632/oncotarget.3055

Chapard C, Meraldi P, Gleich T, Bachmann D, Hohl D, Huber M (2014) TRAIP is a regulator of the spindle assembly checkpoint. J Cell Sci 127: 5149-5156. doi:10.1242/jcs.152579

Dewar JM, Budzowska M, Walter JC (2015) The mechanism of DNA replication termination in vertebrates. Nature 525: 345-350. doi:10.1038/ nature14887

Feng W, Guo Y, Huang J, Deng Y, Zang J, Huen MS (2016) TRAIP regulates replication fork recovery and progression via PCNA. Cell Discov 2: 16016. doi:10.1038/celldisc.2016.16

Fu YV, Yardimci H, Long DT, Ho TV, Guainazzi A, Bermudez VP, Hurwitz J, van Oijen A, Scharer OD, Walter JC (2011) Selective bypass of a lagging strand roadblock by the eukaryotic replicative DNA helicase. Cell 146: 931-941. doi:10.1016/j.cell.2011.07.045

Gambus A, Jones RC, Sanchez-Diaz A, Kanemaki M, van Deursen F, Edmondson RD, Labib K (2006) GINS maintains association of Cdc45 with MCM in replisome progression complexes at eukaryotic DNA replication forks. Nat Cell Biol 8: 358-366. doi:10.1038/ncb1382

Gambus A, Khoudoli GA, Jones RC, Blow JJ (2011) MCM2-7 form double hexamers at licensed origins in Xenopus egg extract. J Biol Chem 286: 11855-11864. doi:10.1074/jbc.m110.199521

Gao Y, Mutter-Rottmayer E, Zlatanou A, Vaziri C, Yang Y (2017) Mechanisms of post-replication DNA repair. Genes (Basel) 8: E64. doi:10.3390/ genes8020064

Gillespie PJ, Gambus A, Blow JJ (2012) Preparation and use of Xenopus egg extracts to study DNA replication and chromatin associated proteins. Methods 57: 203-213. doi:10.1016/j.ymeth.2012.03.029

Gillespie PJ, Khoudoli GA, Stewart G, Swedlow JR, Blow JJ (2007) ELYS/MEL-28 chromatin association coordinates nuclear pore complex assembly and replication licensing. Curr Biol 17: 1657-1662. doi:10.1016/j. cub.2007.08.041

Harley ME, Murina O, Leitch A, Higgs MR, Bicknell LS, Yigit G, Blackford AN, Zlatanou A, Mackenzie KJ, Reddy K, et al (2016) TRAIP promotes DNA damage response during genome replication and is mutated in primordial dwarfism. Nat Genet 48: 36-43. doi:10.1038/ng.3451

Heidelberger JB, Voigt A, Borisova ME, Petrosino G, Ruf S, Wagner SA, Beli P (2018) Proteomic profiling of VCP substrates links VCP to K6-linked 
ubiquitylation and c-Myc function. EMBO Rep 19: e44754. doi:10.15252/ embr.201744754

Hochegger H, Klotzbucher A, Kirk J, Howell M, le Guellec K, Fletcher K, Duncan T, Sohail M, Hunt T (2001) New B-type cyclin synthesis is required between meiosis I and II during Xenopus oocyte maturation. Development 128: 3795-3807.

Hoffmann S, Smedegaard S, Nakamura K, Mortuza GB, Raschle M, Ibanez de Opakua A, Oka Y, Feng Y, Blanco FJ, Mann M, et al (2016) TRAIP is a PCNA-binding ubiquitin ligase that protects genome stability after replication stress. J Cell Biol 212: 63-75. doi:10.1083/jcb.201506071

Kamada F, Aoki Y, Narisawa A, Abe Y, Komatsuzaki S, Kikuchi A, Kanno J, Niihori T, Ono M, Ishii N, et al (2011) A genome-wide association study identifies RNF213 as the first Moyamoya disease gene. J Hum Genet 56: 34-40. doi:10.1038/jhg.2010.132

Khoudoli GA, Gillespie PJ, Stewart G, Andersen JS, Swedlow JR, Blow JJ (2008) Temporal profiling of the chromatin proteome reveals system-wide responses to replication inhibition. Curr Biol 18: 838-843. doi:10.1016/j. cub.2008.04.075

Kobayashi H, Yamazaki S, Takashima S, Liu W, Okuda H, Yan J, Fujii Y, Hitomi T, Harada KH, Habu T, et al (2013) Ablation of Rnf213 retards progression of diabetes in the Akita mouse. Biochem Biophys Res Commun 432: 519-525. doi:10.1016/j.bbrc.2013.02.015

Lee SY, Lee SY, Choi Y (1997) TRAF-interacting protein (TRIP): A novel component of the tumor necrosis factor receptor (TNFR)- and CD30TRAF signaling complexes that inhibits TRAF2-mediated NF-kappaB activation. J Exp Med 185: 1275-1285. doi:10.1084/jem.185.7.1275

Liu W, Morito D, Takashima S, Mineharu Y, Kobayashi H, Hitomi T, Hashikata H, Matsuura N, Yamazaki S, Toyoda A, et al (2011) Identification of RNF213 as a susceptibility gene for moyamoya disease and its possible role in vascular development. PLoS One 6: e22542. doi:10.1371/journal.pone.0022542

Liu Y, Nielsen CF, Yao Q, Hickson ID (2014) The origins and processing of ultra fine anaphase DNA bridges. Curr Opin Genet Dev 26: 1-5. doi:10.1016/j. gde.2014.03.003

Maric M, Maculins T, De Piccoli G, Labib K (2014) Cdc48 and a ubiquitin ligase drive disassembly of the CMG helicase at the end of DNA replication. Science 346: 1253596. doi:10.1126/science.1253596

Maric M, Mukherjee P, Tatham MH, Hay R, Labib K (2017) Ufd1-Npl4 recruit Cdc48 for disassembly of ubiquitylated CMG helicase at the end of chromosome replication. Cell Rep 18: 3033-3042. doi:10.1016/j. celrep.2017.03.020

Meyer H (2012) p97 complexes as signal integration hubs. BMC Biol 10: 48. doi:10.1186/1741-7007-10-48

Meyer H, Bug M, Bremer S (2012) Emerging functions of the VCP/p97 AAAATPase in the ubiquitin system. Nat Cell Biol 14: 117-123. doi:10.1038/ ncb2407

Meyer HJ, Rape M (2014) Enhanced protein degradation by branched ubiquitin chains. Cell 157: 910-921. doi:10.1016/j.cell.2014.03.037

Minocherhomji S, Ying S, Bjerregaard VA, Bursomanno S, Aleliunaite A, Wu W, Mankouri HW, Shen H, Liu Y, Hickson ID (2015) Replication stress activates DNA repair synthesis in mitosis. Nature 528: 286-290. doi:10.1038/nature16139

Minshull J, Blow JJ, Hunt T (1989) Translation of cyclin mRNA is necessary for extracts of activated xenopus eggs to enter mitosis. Cell 56: 947-956. doi:10.1016/0092-8674(89)90628-4

Moreno A, Carrington JT, Albergante L, Al Mamun M, Haagensen EJ, Komseli ES, Gorgoulis VG, Newman TJ, Blow JJ (2016) Unreplicated DNA remaining from unperturbed $S$ phases passes through mitosis for resolution in daughter cells. Proc Natl Acad Sci U S A 113: E5757-E5764. doi:10.1073/ pnas. 1603252113
Moreno SP, Bailey R, Campion N, Herron S, Gambus A (2014)

Polyubiquitylation drives replisome disassembly at the termination of DNA replication. Science 346: 477-481. doi:10.1126/science.1253585

Moyer SE, Lewis PW, Botchan MR (2006) Isolation of the Cdc45/Mcm2-7/GINS (CMG) complex, a candidate for the eukaryotic DNA replication fork helicase. Proc Natl Acad Sci U S A 103: 10236-10241. doi:10.1073/ pnas.0602400103

Park ES, Choi S, Kim JM, Jeong Y, Choe J, Park CS, Choi Y, Rho J (2007) Early embryonic lethality caused by targeted disruption of the TRAFinteracting protein (TRIP) gene. Biochem Biophys Res Commun 363: 971-977. doi:10.1016/j.bbrc.2007.09.103

Park IS, Han Y, Chung HJ, Jung YW, Kim Y, Kim H (2016) SUMOylation regulates nuclear localization and stability of TRAIP/RNF206. Biochem Biophys Res Commun 470: 881-887. doi:10.1016/j.bbrc.2016.01.141

Park IS, Jo KS, Won HS, Kim H (2015) Dimerization of TRAF-interacting protein (TRAIP) regulates the mitotic progression. Biochem Biophys Res Commun 463: 864-869. doi:10.1016/j.bbrc.2015.06.026

Sonneville R, Moreno SP, Knebel A, Johnson C, Hastie CJ, Gartner A, Gambus A, Labib K (2017) CUL-2LRR-1 and UBXN-3 drive replisome disassembly during DNA replication termination and mitosis. Nat Cell Biol 19: 468-479. doi:10.1038/ncb3500

Sonobe S, Fujimura M, Niizuma K, Nishijima Y, Ito A, Shimizu H, Kikuchi A, AraiIchinoi N, Kure S, Tominaga T (2014) Temporal profile of the vascular anatomy evaluated by 9.4-T magnetic resonance angiography and histopathological analysis in mice lacking RNF213: A susceptibility gene for moyamoya disease. Brain Res 1552: 64-71. doi:10.1016/j. brainres.2014.01.011

Soo Lee N, Jin Chung H, Kim HJ, Yun Lee S, Ji JH, Seo Y, Hun Han S, Choi M, Yun M, Lee SG, et al (2016) TRAIP/RNF206 is required for recruitment of RAP80 to sites of DNA damage. Nat Commun 7: 10463. doi:10.1038/ ncomms10463

Strausfeld UP, Howell M, Descombes P, Chevalier S, Rempel RE, Adamczewski J, Maller JL, Hunt T, Blow JJ (1996) Both cyclin A and cyclin E have Sphase promoting (SPF) activity in Xenopus egg extracts. J Cell Sci 109: 1555-1563.

Wallace HA, Merkle JA, Yu MC, Berg TG, Lee E, Bosco G, Lee LA (2014) TRIP/ NOPO E3 ubiquitin ligase promotes ubiquitylation of DNA polymerase eta. Development 141: 1332-1341. doi:10.1242/dev.101196

Windheim M, Peggie M, Cohen P (2008) Two different classes of E2 ubiquitinconjugating enzymes are required for the mono-ubiquitination of proteins and elongation by polyubiquitin chains with a specific topology. Biochem J 409: 723-729. doi:10.1042/bj20071338

Wu RA, Semlow DR, Kamimae-Lanning AN, Kochenova OV, Chistol G, Hodskinson MR, Amunugama R, Sparks JL, Wang M, Deng L, et al (2019) TRAIP is a master regulator of DNA interstrand crosslink repair. Nature 567: 267-272. doi:10.1038/s41586-019-1002-0

Yang C, Trent S, Ionescu-Tiba V, Lan L, Shioda T, Sgroi D, Schmidt EV (2006) Identification of cyclin D1- and estrogen-regulated genes contributing to breast carcinogenesis and progression. Cancer Res 66: 11649-11658. doi:10.1158/0008-5472.can-06-1645

Zhou Q, Geahlen RL (2009) The protein-tyrosine kinase Syk interacts with TRAF-interacting protein TRIP in breast epithelial cells. Oncogene 28: 1348-1356. doi:10.1038/onc.2008.493

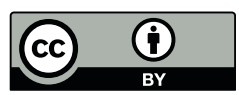

License: This article is available under a Creative Commons License (Attribution 4.0 International, as described at https://creativecommons.org/ licenses/by/4.0/). 